\title{
Existence of Solutions to Nonlinear Langevin Equation Involving Two Fractional Orders with Boundary Value Conditions
}

\author{
Anping Chen ${ }^{1,2}$ and Yi Chen ${ }^{2}$ \\ ${ }^{1}$ Department of Mathematics, Xiangnan University, Chenzhou, Hunan 423000, China \\ ${ }^{2}$ School of Mathematics and Computational Science, Xiangtan University, Xiangtan, Hunan 411005, China
}

Correspondence should be addressed to Anping Chen, chenap@263.net

Received 30 September 2010; Revised 21 January 2011; Accepted 26 February 2011

Academic Editor: Kanishka Perera

Copyright (C) 2011 A. Chen and Y. Chen. This is an open access article distributed under the Creative Commons Attribution License, which permits unrestricted use, distribution, and reproduction in any medium, provided the original work is properly cited.

We study a boundary value problem to Langevin equation involving two fractional orders. The Banach fixed point theorem and Krasnoselskii's fixed point theorem are applied to establish the existence results.

\section{Introduction}

Recently, the subject of fractional differential equations has emerged as an important area of investigation. Indeed, we can find numerous applications in viscoelasticity, electrochemistry, control, electromagnetic, porous media, and so forth. In consequence, the subject of fractional differential equations is gaining much importance and attention. For some recent developments on the subject, see [1-8] and the references therein.

Langevin equation is widely used to describe the evolution of physical phenomena in fluctuating environments. However, for systems in complex media, ordinary Langevin equation does not provide the correct description of the dynamics. One of the possible generalizations of Langevin equation is to replace the ordinary derivative by a fractional derivative in it. This gives rise to fractional Langevin equation, see for instance [9-12] and the references therein.

In this paper, we consider the following boundary value problem of Langevin equation with two different fractional orders:

$$
\begin{gathered}
{ }^{C} D^{\beta}\left({ }^{C} D^{\alpha}+\lambda\right) u(t)=f(t, u(t)) \quad t \in[0, T], \\
u(0)=-u(T), \quad u^{\prime}(0)=u^{\prime}(T)=0,
\end{gathered}
$$


where $T$ is a positive constant, $1<\alpha \leq 2,0<\beta \leq 1,{ }^{C} D^{\alpha}$, and ${ }^{C} D^{\beta}$ are the Caputo fractional derivatives, $f:[0, T] \times R \rightarrow R$ is continuous, and $\lambda$ is a real number.

The organization of this paper is as follows. In Section 2, we recall some definitions of fractional integral and derivative and preliminary results which will be used in this paper. In Section 3, we will consider the existence results for problem (1.1). In Section 4, we will give an example to ensure our main results.

\section{Preliminaries}

In this section, we present some basic notations, definitions, and preliminary results which will be used throughout this paper.

Definition 2.1. The Caputo fractional derivative of order $\alpha$ of a function $f:[0, \infty) \rightarrow R$, is defined as

$$
{ }^{C} D^{\alpha} f(t)=\frac{1}{\Gamma(n-\alpha)} \int_{0}^{t}(t-s)^{n-\alpha-1} f^{(n)}(s) d s, \quad n-1<\alpha<n, n=[\alpha]+1,
$$

where $[\alpha]$ denotes the integer part of the real number $\alpha$.

Definition 2.2. The Riemann-Liouville fractional integral of order $\alpha>0$ of a function $f(t)$, $t>0$, is defined as

$$
I^{\alpha} f(t)=\frac{1}{\Gamma(\alpha)} \int_{0}^{t}(t-s)^{\alpha-1} f(s) d s,
$$

provided that the right side is pointwise defined on $(0, \infty)$.

Definition 2.3. The Riemann-Liouville fractional derivative of order $\alpha>0$ of a continuous function $f:(0, \infty) \rightarrow R$ is given by

$$
D^{\alpha} f(t)=\frac{1}{\Gamma(n-\alpha)}\left(\frac{d}{d t}\right)^{n} \int_{0}^{t}(t-s)^{n-\alpha-1} f(s) d s,
$$

where $n=[\alpha]+1$ and $[\alpha]$ denotes the integer part of real number $\alpha$, provided that the right side is pointwise defined on $(0, \infty)$.

Lemma 2.4 (see [8]). Let $\alpha>0$, then the fractional differential equation ${ }^{C} D^{\alpha} u(t)=0$ has solution

$$
u(t)=c_{0}+c_{1} t+c_{2} t^{2}+\cdots+c_{n-1} t^{n-1},
$$

where $c_{i} \in R, i=0,1,2, \ldots, n-1, n=[\alpha]+1$.

Lemma 2.5 (see [8]). Let $\alpha>0$, then

$$
I^{\alpha C} D^{\alpha} u(t)=u(t)+c_{0}+c_{1} t+c_{2} t^{2}+\cdots+c_{n-1} t^{n-1},
$$

for some $c_{i} \in R, i=0,1,2, \ldots, n-1, n=[\alpha]+1$. 
Lemma 2.6. The unique solution of the following boundary value problem

$$
\begin{gathered}
{ }^{C} D^{\beta}\left({ }^{C} D^{\alpha}+\lambda\right) u(t)=y(t), \quad t \in[0, T], 1<\alpha \leq 2,0<\beta \leq 1, \\
u(0)=-u(T), \quad u^{\prime}(0)=u^{\prime}(T)=0,
\end{gathered}
$$

is given by

$$
\begin{aligned}
u(t)= & \int_{0}^{t} \frac{(t-s)^{\alpha-1}}{\Gamma(\alpha)}\left(\int_{0}^{s} \frac{(s-\tau)^{\beta-1}}{\Gamma(\beta)} y(\tau) d \tau-\lambda u(s)\right) d s \\
& -\frac{1}{2} \int_{0}^{T} \frac{(T-s)^{\alpha-1}}{\Gamma(\alpha)}\left(\int_{0}^{s} \frac{(s-\tau)^{\beta-1}}{\Gamma(\beta)} y(\tau) d \tau-\lambda u(s)\right) d s \\
& +\frac{T^{\alpha}-2 t^{\alpha}}{2 \alpha T^{\alpha-1}} \int_{0}^{T} \frac{(T-s)^{\alpha-2}}{\Gamma(\alpha-1)}\left(\int_{0}^{s} \frac{(s-\tau)^{\beta-1}}{\Gamma(\beta)} y(\tau) d \tau-\lambda u(s)\right) d s .
\end{aligned}
$$

Proof. Similar to the discussion of [9, equation (1.5)], the general solution of

$$
{ }^{C} D^{\beta}\left({ }^{C} D^{\alpha}+\lambda\right) u(t)=y(t)
$$

can be written as

$$
u(t)=\int_{0}^{t} \frac{(t-s)^{\alpha-1}}{\Gamma(\alpha)}\left(\int_{0}^{s} \frac{(s-\tau)^{\beta-1}}{\Gamma(\beta)} y(\tau) d \tau-\lambda u(s)\right) d s-\frac{c_{0}}{\Gamma(\alpha+1)} t^{\alpha}-c_{1} t-c_{2}
$$

By the boundary conditions $u(0)+u(T)=0$ and $u^{\prime}(0)=u^{\prime}(T)=0$, we obtain

$$
\begin{aligned}
\frac{c_{0}}{\Gamma(\alpha+1)}= & \frac{1}{\alpha T^{\alpha-1}} \int_{0}^{T} \frac{(T-s)^{\alpha-2}}{\Gamma(\alpha-1)}\left(\int_{0}^{s} \frac{(s-\tau)^{\beta-1}}{\Gamma(\beta)} y(\tau) d \tau-\lambda u(s)\right) d s, \\
c_{1}= & 0, \\
c_{2}= & \frac{1}{2} \int_{0}^{T} \frac{(T-s)^{\alpha-1}}{\Gamma(\alpha)}\left(\int_{0}^{s} \frac{(s-\tau)^{\beta-1}}{\Gamma(\beta)} y(\tau) d \tau-\lambda u(s)\right) d s \\
& -\frac{T}{2 \alpha} \int_{0}^{T} \frac{(T-s)^{\alpha-2}}{\Gamma(\alpha-1)}\left(\int_{0}^{s} \frac{(s-\tau)^{\beta-1}}{\Gamma(\beta)} y(\tau) d \tau-\lambda u(s)\right) d s .
\end{aligned}
$$


Hence,

$$
\begin{aligned}
u(t)= & \int_{0}^{t} \frac{(t-s)^{\alpha-1}}{\Gamma(\alpha)}\left(\int_{0}^{s} \frac{(s-\tau)^{\beta-1}}{\Gamma(\beta)} y(\tau) d \tau-\lambda u(s)\right) d s \\
& -\frac{1}{2} \int_{0}^{T} \frac{(T-s)^{\alpha-1}}{\Gamma(\alpha)}\left(\int_{0}^{s} \frac{(s-\tau)^{\beta-1}}{\Gamma(\beta)} y(\tau) d \tau-\lambda u(s)\right) d s \\
& +\frac{T^{\alpha}-2 t^{\alpha}}{2 \alpha T^{\alpha-1}} \int_{0}^{T} \frac{(T-s)^{\alpha-2}}{\Gamma(\alpha-1)}\left(\int_{0}^{s} \frac{(s-\tau)^{\beta-1}}{\Gamma(\beta)} y(\tau) d \tau-\lambda \mathcal{u}(s)\right) d s .
\end{aligned}
$$

Lemma 2.7 (Krasnoselskii's fixed point theorem). Let E be a bounded closed convex subset of a Banach space $X$, and let $S, T$ be the operators such that

(i) $S u+T v \in E$ whenever $u, v \in E$,

(ii) $S$ is completely continuous,

(iii) $T$ is a contraction mapping.

Then there exists $z \in E$ such that $z=S z+T z$.

Lemma 2.8 (Hölder inequality). Let $p>1,(1 / p)+(1 / q)=1, f \in L^{p}[a, b], g \in L^{q}[a, b]$, then the following inequality holds:

$$
\int_{a}^{b}|f(x) g(x)| d x \leq\left[\int_{a}^{b}|f(x)|^{p} d x\right]^{1 / p}\left[\int_{a}^{b}|g(x)|^{q} d x\right]^{1 / q} .
$$

\section{Main Result}

In this section, our aim is to discuss the existence and uniqueness of solutions to the problem (1.1).

Let $\Omega$ be a Banach space of all continuous functions from $[0, T] \rightarrow R$ with the norm $\|u\|=\sup _{t \in[0, T]}\{|u(t)|\}$.

Theorem 3.1. Assume that

(H1) there exists a real-valued function $\mu(t) \in L^{1 / \gamma}\left([0, T], R^{+}\right)$for some $\gamma \in(0,1)$ such that

$$
|f(t, u)-f(t, v)| \leq \mu(t)|u-v|, \quad \text { for almost all } t \in[0, T], u, v \in R
$$

If

$$
\Lambda \triangleq \frac{(4 \alpha+\beta-\gamma) \Gamma(\beta-\gamma+1) \mu^{*} T^{\alpha+\beta-\gamma}}{2 \alpha \Gamma(\beta) \Gamma(\alpha+\beta-\gamma+1)}\left(\frac{1-\gamma}{\beta-\gamma}\right)^{1-\gamma}+\frac{2|\lambda| T^{\alpha}}{\Gamma(\alpha+1)}<1
$$

where $r \in(0,1), \beta \neq \gamma, 1<\alpha \leq 2,0<\beta \leq 1, \mu^{*}=\left(\int_{0}^{T}(\mu(\tau))^{1 / \gamma} d \tau\right)^{\gamma}$, then problem (1.1) has a unique solution. 
Proof. Define an operator $F: \Omega \rightarrow \Omega$ by

$$
\begin{aligned}
(F u)(t)= & \int_{0}^{t} \frac{(t-s)^{\alpha-1}}{\Gamma(\alpha)}\left(\int_{0}^{s} \frac{(s-\tau)^{\beta-1}}{\Gamma(\beta)} f(\tau, u(\tau)) d \tau-\lambda u(s)\right) d s \\
& -\frac{1}{2} \int_{0}^{T} \frac{(T-s)^{\alpha-1}}{\Gamma(\alpha)}\left(\int_{0}^{s} \frac{(s-\tau)^{\beta-1}}{\Gamma(\beta)} f(\tau, u(\tau)) d \tau-\lambda u(s)\right) d s \\
& +\frac{T^{\alpha}-2 t^{\alpha}}{2 \alpha T^{\alpha-1}} \int_{0}^{T} \frac{(T-s)^{\alpha-2}}{\Gamma(\alpha-1)}\left(\int_{0}^{s} \frac{(s-\tau)^{\beta-1}}{\Gamma(\beta)} f(\tau, u(\tau)) d \tau-\lambda \mathcal{u}(s)\right) d s .
\end{aligned}
$$

Let $M=\sup _{t \in[0, T]}|f(t, 0)|$ and choose

$$
\frac{1}{1-\delta}\left(\frac{(4 \alpha+\beta) M T^{\alpha+\beta}}{2 \alpha \Gamma(\alpha+\beta+1)}\right) \leq r
$$

where $\delta$ is such that $\Lambda \leq \delta<1$.

Now we show that $F B_{r} \subset B_{r}$, where $B_{r}=\{u \in \Omega:\|u\| \leq r\}$. For $u \in B_{r}$, by Hölder inequality, we have

$$
\begin{aligned}
& |(F u)(t)| \\
& =\mid \int_{0}^{t} \frac{(t-s)^{\alpha-1}}{\Gamma(\alpha)}\left(\int_{0}^{s} \frac{(s-\tau)^{\beta-1}}{\Gamma(\beta)} f(\tau, u(\tau)) d \tau-\lambda u(s)\right) d s \\
& \quad-\frac{1}{2} \int_{0}^{T} \frac{(T-s)^{\alpha-1}}{\Gamma(\alpha)}\left(\int_{0}^{s} \frac{(s-\tau)^{\beta-1}}{\Gamma(\beta)} f(\tau, u(\tau)) d \tau-\lambda u(s)\right) d s \\
& \quad+\frac{T^{\alpha}-2 t^{\alpha}}{2 \alpha T^{\alpha-1}} \int_{0}^{T} \frac{(T-s)^{\alpha-2}}{\Gamma(\alpha-1)}\left(\int_{0}^{s} \frac{(s-\tau)^{\beta-1}}{\Gamma(\beta)} f(\tau, u(\tau)) d \tau-\lambda u(s)\right) d s \mid \\
& \leq \int_{0}^{t} \frac{(t-s)^{\alpha-1}}{\Gamma(\alpha)}\left(\int_{0}^{s} \frac{(s-\tau)^{\beta-1}}{\Gamma(\beta)}((|f(\tau, u(\tau))-f(\tau, 0)|)+|f(\tau, 0)|) d \tau+|\lambda u(s)|\right) d s \\
& \quad+\frac{1}{2} \int_{0}^{T} \frac{(T-s)^{\alpha-1}}{\Gamma(\alpha)}\left(\int_{0}^{s} \frac{(s-\tau)^{\beta-1}}{\Gamma(\beta)}((|f(\tau, u(\tau))-f(\tau, 0)|)+|f(\tau, 0)|) d \tau+|\lambda u(s)|\right) d s \\
& \quad+\frac{T}{2 \alpha} \int_{0}^{T} \frac{(T-s)^{\alpha-2}}{\Gamma(\alpha-1)}\left(\int_{0}^{s} \frac{(s-\tau)^{\beta-1}}{\Gamma(\beta)}((|f(\tau, u(\tau))-f(\tau, 0)|)+|f(\tau, 0)|) d \tau+|\lambda u(s)|\right) d s \\
& \leq \int_{0}^{t} \frac{(t-s)^{\alpha-1}}{\Gamma(\alpha)}\left(\int_{0}^{s} \frac{(s-\tau)^{\beta-1}}{\Gamma(\beta)}(\mu(\tau)|u(\tau)|+|f(\tau, 0)|) d \tau+|\lambda u(s)|\right) d s \\
& \quad+\frac{1}{2} \int_{0}^{T} \frac{(T-s)^{\alpha-1}}{\Gamma(\alpha)}\left(\int_{0}^{s} \frac{(s-\tau)^{\beta-1}}{\Gamma(\beta)}(\mu(\tau)|u(\tau)|+|f(\tau, 0)|) d \tau+|\mathcal{\lambda} u(s)|\right) d s
\end{aligned}
$$




$$
\begin{aligned}
& +\frac{T}{2 \alpha} \int_{0}^{T} \frac{(T-s)^{\alpha-2}}{\Gamma(\alpha-1)}\left(\int_{0}^{s} \frac{(s-\tau)^{\beta-1}}{\Gamma(\beta)}(\mu(\tau)|u(\tau)|+|f(\tau, 0)|) d \tau+|\lambda u(s)|\right) d s \\
& \leq\|u\| \int_{0}^{t} \frac{(t-s)^{\alpha-1}}{\Gamma(\alpha)}\left(\int_{0}^{s} \frac{(s-\tau)^{\beta-1}}{\Gamma(\beta)} \mu(\tau) d \tau\right) d s+M \int_{0}^{t} \frac{(t-s)^{\alpha-1}}{\Gamma(\alpha)} \int_{0}^{s} \frac{(s-\tau)^{\beta-1}}{\Gamma(\beta)} d \tau d s \\
& +|\lambda|\|u\| \int_{0}^{t} \frac{(t-s)^{\alpha-1}}{\Gamma(\alpha)} d s+\frac{\|u\|}{2} \int_{0}^{T} \frac{(T-s)^{\alpha-1}}{\Gamma(\alpha)}\left(\int_{0}^{s} \frac{(s-\tau)^{\beta-1}}{\Gamma(\beta)} \mu(\tau) d \tau\right) d s \\
& +\frac{M}{2} \int_{0}^{T} \frac{(T-s)^{\alpha-1}}{\Gamma(\alpha)} \int_{0}^{s} \frac{(s-\tau)^{\beta-1}}{\Gamma(\beta)} d \tau d s+\frac{|\lambda|\|u\|}{2} \int_{0}^{T} \frac{(T-s)^{\alpha-1}}{\Gamma(\alpha)} d s \\
& +\frac{T\|u\|}{2 \alpha} \int_{0}^{T} \frac{(T-s)^{\alpha-2}}{\Gamma(\alpha-1)}\left(\int_{0}^{s} \frac{(s-\tau)^{\beta-1}}{\Gamma(\beta)} \mu(\tau) d \tau\right) d s \\
& +\frac{T M}{2 \alpha} \int_{0}^{T} \frac{(T-s)^{\alpha-2}}{\Gamma(\alpha-1)} \int_{0}^{s} \frac{(s-\tau)^{\beta-1}}{\Gamma(\beta)} d \tau d s+\frac{T|\lambda|\|u\|}{2 \alpha} \int_{0}^{T} \frac{(T-s)^{\alpha-2}}{\Gamma(\alpha-1)} d s \\
& \leq \frac{\|u\|}{\Gamma(\alpha) \Gamma(\beta)} \int_{0}^{t}(t-s)^{\alpha-1}\left[\left(\int_{0}^{s}\left((s-\tau)^{\beta-1}\right)^{1 /(1-\gamma)} d \tau\right)^{1-\gamma}\left(\int_{0}^{s}(\mu(\tau))^{1 / \gamma} d \tau\right)^{\gamma}\right] d s \\
& +\frac{M}{\Gamma(\alpha) \Gamma(\beta+1)} \int_{0}^{t}(t-s)^{\alpha-1} s^{\beta} d s+\frac{|\lambda| T^{\alpha}\|u\|}{\Gamma(\alpha+1)} \\
& +\frac{\|u\|}{2 \Gamma(\alpha) \Gamma(\beta)} \int_{0}^{T}(T-s)^{\alpha-1}\left[\left(\int_{0}^{s}\left((s-\tau)^{\beta-1}\right)^{1 /(1-\gamma)} d \tau\right)^{1-\gamma}\left(\int_{0}^{s}(\mu(\tau))^{1 / \gamma} d \tau\right)^{\gamma}\right] d s \\
& +\frac{M}{2 \Gamma(\alpha) \Gamma(\beta+1)} \int_{0}^{T}(T-s)^{\alpha-1} s^{\beta} d s+\frac{|\lambda| T^{\alpha}\|u\|}{2 \Gamma(\alpha+1)} \\
& +\frac{T\|u\|}{2 \alpha \Gamma(\alpha-1) \Gamma(\beta)} \int_{0}^{T}(T-s)^{\alpha-2}\left[\left(\int_{0}^{s}\left((s-\tau)^{\beta-1}\right)^{1 /(1-\gamma)} d \tau\right)^{1-\gamma}\left(\int_{0}^{s}(\mu(\tau))^{1 / \gamma} d \tau\right)^{\gamma}\right] d s \\
& +\frac{T M}{2 \alpha \Gamma(\alpha-1) \Gamma(\beta+1)} \int_{0}^{T}(T-s)^{\alpha-2} s^{\beta} d s+\frac{|\lambda| T^{\alpha}\|u\|}{2 \Gamma(\alpha+1)} \\
& \leq \frac{\mu^{*}\|u\|}{\Gamma(\alpha) \Gamma(\beta)}\left(\frac{1-\gamma}{\beta-\gamma}\right)^{1-\gamma} \int_{0}^{t}(t-s)^{\alpha-1} s^{\beta-\gamma} d s+\frac{M}{\Gamma(\alpha) \Gamma(\beta+1)} \int_{0}^{t}(t-s)^{\alpha-1} s^{\beta} d s \\
& +\frac{\mu^{*}\|u\|}{2 \Gamma(\alpha) \Gamma(\beta)}\left(\frac{1-\gamma}{\beta-\gamma}\right)^{1-\gamma} \int_{0}^{T}(T-s)^{\alpha-1} s^{\beta-\gamma} d s+\frac{M}{2 \Gamma(\alpha) \Gamma(\beta+1)} \int_{0}^{T}(T-s)^{\alpha-1} s^{\beta} d s \\
& +\frac{T \mu^{*}\|u\|}{2 \alpha \Gamma(\alpha-1) \Gamma(\beta)}\left(\frac{1-\gamma}{\beta-\gamma}\right)^{1-\gamma} \int_{0}^{T}(T-s)^{\alpha-2} s^{\beta-\gamma} d s \\
& +\frac{T M}{2 \alpha \Gamma(\alpha-1) \Gamma(\beta+1)} \int_{0}^{T}(T-s)^{\alpha-2} s^{\beta} d s+\frac{2|\lambda| T^{\alpha}\|u\|}{\Gamma(\alpha+1)}
\end{aligned}
$$


Boundary Value Problems

$$
\begin{aligned}
= & \frac{\mu^{*}\|u\| t^{\alpha+\beta-\gamma}}{\Gamma(\alpha) \Gamma(\beta)}\left(\frac{1-\gamma}{\beta-\gamma}\right)^{1-\gamma} \int_{0}^{1}(1-\xi)^{\alpha-1} \xi^{\beta-\gamma} d \xi+\frac{M t^{\alpha+\beta}}{\Gamma(\alpha) \Gamma(\beta+1)} \int_{0}^{1}(1-\xi)^{\alpha-1} \xi^{\beta} d \xi \\
& +\frac{\mu^{*}\|u\| T^{\alpha+\beta-\gamma}}{2 \Gamma(\alpha) \Gamma(\beta)}\left(\frac{1-\gamma}{\beta-\gamma}\right)^{1-\gamma} \int_{0}^{1}(1-\eta)^{\alpha-1} \eta^{\beta-\gamma} d \eta+\frac{M T^{\alpha+\beta}}{2 \Gamma(\alpha) \Gamma(\beta+1)} \int_{0}^{1}(1-\eta)^{\alpha-1} \eta^{\beta} d \eta \\
& +\frac{\mu^{*}\|u\| T^{\alpha+\beta-\gamma}}{2 \alpha \Gamma(\alpha-1) \Gamma(\beta)}\left(\frac{1-\gamma}{\beta-\gamma}\right)^{1-\gamma} \int_{0}^{1}(1-\eta)^{\alpha-2} \eta^{\beta-\gamma} d \eta \\
& +\frac{M T^{\alpha+\beta}}{2 \alpha \Gamma(\alpha-1) \Gamma(\beta+1)} \int_{0}^{1}(1-\eta)^{\alpha-2} \eta^{\beta} d \eta+\frac{2|\lambda| T^{\alpha}\|u\|}{\Gamma(\alpha+1)} \\
\leq & \frac{r \mu^{*} T^{\alpha+\beta-\gamma}}{\Gamma(\alpha) \Gamma(\beta)}\left(\frac{1-\gamma}{\beta-\gamma}\right)^{1-\gamma} \int_{0}^{1}(1-\xi)^{\alpha-1} \xi^{\beta-\gamma} d \xi+\frac{M T^{\alpha+\beta}}{\Gamma(\alpha) \Gamma(\beta+1)} \int_{0}^{1}(1-\xi)^{\alpha-1} \xi^{\beta} d \xi \\
& +\frac{r \mu^{*} T^{\alpha+\beta-\gamma}}{2 \Gamma(\alpha) \Gamma(\beta)}\left(\frac{1-\gamma}{\beta-\gamma}\right)^{1-\gamma} \int_{0}^{1}(1-\eta)^{\alpha-1} \eta^{\beta-\gamma} d \eta+\frac{M T^{\alpha+\beta}}{2 \Gamma(\alpha) \Gamma(\beta+1)} \int_{0}^{1}(1-\eta)^{\alpha-1} \eta^{\beta} d \eta \\
& +\frac{r \mu^{*} T^{\alpha+\beta-\gamma}}{2 \alpha \Gamma(\alpha-1) \Gamma(\beta)}\left(\frac{1-\gamma}{\beta-\gamma}\right)^{1-\gamma} \int_{0}^{1}(1-\eta)^{\alpha-2} \eta^{\beta-\gamma} d \eta \\
& +\frac{M T^{\alpha+\beta}}{2 \alpha \Gamma(\alpha-1) \Gamma(\beta+1)} \int_{0}^{1}(1-\eta)^{\alpha-2} \eta^{\beta} d \eta+\frac{2|\lambda| T^{\alpha} r}{\Gamma(\alpha+1)} .
\end{aligned}
$$

Take notice of Beta functions:

$$
\begin{aligned}
B(\beta-\gamma+1, \alpha) & =\int_{0}^{1}(1-\xi)^{\alpha-1} \xi^{\beta-\gamma} d \xi=\int_{0}^{1}(1-\eta)^{\alpha-1} \eta^{\beta-\gamma} d \eta=\frac{\Gamma(\alpha) \Gamma(\beta-\gamma+1)}{\Gamma(\alpha+\beta-\gamma+1)} \\
B(\beta+1, \alpha) & =\int_{0}^{1}(1-\xi)^{\alpha-1} \xi^{\beta} d \xi=\int_{0}^{1}(1-\eta)^{\alpha-1} \eta^{\beta} d \eta=\frac{\Gamma(\alpha) \Gamma(\beta+1)}{\Gamma(\alpha+\beta+1)} \\
B(\beta-\gamma+1, \alpha-1) & =\int_{0}^{1}(1-\eta)^{\alpha-2} \eta^{\beta-\gamma} d \eta=\frac{\Gamma(\alpha-1) \Gamma(\beta-\gamma+1)}{\Gamma(\alpha+\beta-\gamma)} \\
B(\beta+1, \alpha-1) & =\int_{0}^{1}(1-\eta)^{\alpha-2} \eta^{\beta} d \eta=\frac{\Gamma(\alpha-1) \Gamma(\beta+1)}{\Gamma(\alpha+\beta)} .
\end{aligned}
$$

We can get

$$
\begin{aligned}
|(F u)(t)| \leq & \frac{r \mu^{*} \Gamma(\beta-\gamma+1) T^{\alpha+\beta-\gamma}}{\Gamma(\beta) \Gamma(\alpha+\beta-\gamma+1)}\left(\frac{1-\gamma}{\beta-\gamma}\right)^{1-\gamma}+\frac{M T^{\alpha+\beta}}{\Gamma(\alpha+\beta+1)} \\
& +\frac{r \mu^{*} \Gamma(\beta-\gamma+1) T^{\alpha+\beta-\gamma}}{2 \Gamma(\beta) \Gamma(\alpha+\beta-\gamma+1)}\left(\frac{1-\gamma}{\beta-\gamma}\right)^{1-\gamma}+\frac{M T^{\alpha+\beta}}{2 \Gamma(\alpha+\beta+1)}
\end{aligned}
$$




$$
\begin{aligned}
& +\frac{r \mu^{*} \Gamma(\beta-\gamma+1) T^{\alpha+\beta-\gamma}}{2 \alpha \Gamma(\beta) \Gamma(\alpha+\beta-\gamma)}\left(\frac{1-\gamma}{\beta-\gamma}\right)^{1-\gamma}+\frac{M T^{\alpha+\beta}}{2 \alpha \Gamma(\alpha+\beta)}+\frac{2|\lambda| T^{\alpha} r}{\Gamma(\alpha+1)} \\
= & {\left[\frac{(4 \alpha+\beta-\gamma) \Gamma(\beta-\gamma+1) \mu^{*} T^{\alpha+\beta-\gamma}}{2 \alpha \Gamma(\beta) \Gamma(\alpha+\beta-\gamma+1)}\left(\frac{1-\gamma}{\beta-\gamma}\right)^{1-\gamma}+\frac{2|\lambda| T^{\alpha}}{\Gamma(\alpha+1)}\right] r } \\
& +\frac{(4 \alpha+\beta) M T^{\alpha+\beta}}{2 \alpha \Gamma(\alpha+\beta+1)} \\
\leq & (\Lambda+1-\delta) r \\
\leq & r .
\end{aligned}
$$

Therefore, $\|(F u)(t)\| \leq r$.

For $u, v \in \Omega$ and for each $t \in[0, T]$, based on Hölder inequality, we obtain

$$
\begin{aligned}
& |(F u)(t)-(F v)(t)| \\
& \leq \int_{0}^{t} \frac{(t-s)^{\alpha-1}}{\Gamma(\alpha)}\left(\int_{0}^{s} \frac{(s-\tau)^{\beta-1}}{\Gamma(\beta)}|f(\tau, u(\tau))-f(\tau, v(\tau))| d \tau\right) d s \\
& \quad+|\lambda| \int_{0}^{t} \frac{(t-s)^{\alpha-1}}{\Gamma(\alpha)}|u(s)-v(s)| d s \\
& \quad+\frac{1}{2} \int_{0}^{T} \frac{(T-s)^{\alpha-1}}{\Gamma(\alpha)}\left(\int_{0}^{s} \frac{(s-\tau)^{\beta-1}}{\Gamma(\beta)}|f(\tau, u(\tau))-f(\tau, v(\tau))| d \tau\right) d s \\
& \quad+\frac{|\lambda|}{2} \int_{0}^{T} \frac{(T-s)^{\alpha-1}}{\Gamma(\alpha)}|u(s)-v(s)| d s \\
& \quad+\frac{T}{2 \alpha} \int_{0}^{T} \frac{(T-s)^{\alpha-2}}{\Gamma(\alpha-1)}\left(\int_{0}^{s} \frac{(s-\tau)^{\beta-1}}{\Gamma(\beta)}|f(\tau, u(\tau))-f(\tau, v(\tau))| d \tau\right) d s \\
& \quad+\frac{|\lambda| T}{2 \alpha} \int_{0}^{T} \frac{(T-s)^{\alpha-2}}{\Gamma(\alpha-1)}|u(s)-v(s)| d s \\
& \leq \frac{\|u-v\|}{\Gamma(\alpha) \Gamma(\beta)} \int_{0}^{t}(t-s)^{\alpha-1}\left(\int_{0}^{s}(s-\tau)^{\beta-1} \mu(\tau) d \tau\right) d s+\frac{|\lambda| T^{\alpha}}{\Gamma(\alpha+1)}\|u-v\| \\
& \quad+\frac{\|u-v\|}{2 \Gamma(\alpha) \Gamma(\beta)} \int_{0}^{T}(T-s)^{\alpha-1}\left(\int_{0}^{s}(s-\tau)^{\beta-1} \mu(\tau) d \tau\right) d s+\frac{|\lambda| T^{\alpha}}{2 \Gamma(\alpha+1)}\|u-v\| \\
& \quad+\frac{T\|u-v\|}{2 \alpha \Gamma(\alpha-1) \Gamma(\beta)} \int_{0}^{T}(T-s)^{\alpha-2}\left(\int_{0}^{s}(s-\tau)^{\beta-1} \mu(\tau) d \tau\right) d s+\frac{|\lambda| T^{\alpha}}{2 \Gamma(\alpha+1)}\|u-v\|
\end{aligned}
$$




$$
\begin{aligned}
& \leq \frac{\|u-v\|}{\Gamma(\alpha) \Gamma(\beta)} \int_{0}^{t}(t-s)^{\alpha-1}\left[\left(\int_{0}^{s}\left((s-\tau)^{\beta-1}\right)^{1 /(1-\gamma)} d \tau\right)^{1-\gamma}\left(\int_{0}^{s}(\mu(\tau))^{1 / \gamma} d \tau\right)^{\gamma}\right] d s \\
& +\frac{\|u-v\|}{2 \Gamma(\alpha) \Gamma(\beta)} \int_{0}^{T}(T-s)^{\alpha-1}\left[\left(\int_{0}^{s}\left((s-\tau)^{\beta-1}\right)^{1 /(1-\gamma)} d \tau\right)^{1-\gamma}\left(\int_{0}^{s}(\mu(\tau))^{1 / \gamma} d \tau\right)^{\gamma}\right] d s \\
& +\frac{T\|u-v\|}{2 \alpha \Gamma(\alpha-1) \Gamma(\beta)} \int_{0}^{T}(T-s)^{\alpha-2}\left[\left(\int_{0}^{s}\left((s-\tau)^{\beta-1}\right)^{1 /(1-\gamma)} d \tau\right)^{1-\gamma}\left(\int_{0}^{s}(\mu(\tau))^{1 / \gamma} d \tau\right)^{\gamma}\right] d s \\
& +\frac{2|\lambda| T^{\alpha}}{\Gamma(\alpha+1)}\|u-v\| \\
& \leq \frac{\mu^{*}\|u-v\|}{\Gamma(\alpha) \Gamma(\beta)}\left(\frac{1-\gamma}{\beta-\gamma}\right)^{1-\gamma} \int_{0}^{t}(t-s)^{\alpha-1} s^{\beta-\gamma} d s \\
& +\frac{\mu^{*}\|u-v\|}{2 \Gamma(\alpha) \Gamma(\beta)}\left(\frac{1-\gamma}{\beta-\gamma}\right)^{1-\gamma} \int_{0}^{T}(T-s)^{\alpha-1} s^{\beta-\gamma} d s \\
& +\frac{\mu^{*} T\|u-v\|}{2 \alpha \Gamma(\alpha-1) \Gamma(\beta)}\left(\frac{1-\gamma}{\beta-\gamma}\right)^{1-\gamma} \int_{0}^{T}(T-s)^{\alpha-2} s^{\beta-\gamma} d s+\frac{2|\lambda| T^{\alpha}}{\Gamma(\alpha+1)}\|u-v\| \\
& =\frac{\mu^{*}\|u-v\| t^{\alpha+\beta-\gamma}}{\Gamma(\alpha) \Gamma(\beta)}\left(\frac{1-\gamma}{\beta-\gamma}\right)^{1-\gamma} \int_{0}^{1}(1-\xi)^{\alpha-1} \xi^{\beta-\gamma} d \xi \\
& +\frac{\mu^{*}\|u-v\| T^{\alpha+\beta-\gamma}}{2 \Gamma(\alpha) \Gamma(\beta)}\left(\frac{1-\gamma}{\beta-\gamma}\right)^{1-\gamma} \int_{0}^{1}(1-\eta)^{\alpha-1} \eta^{\beta-\gamma} d \eta \\
& +\frac{\mu^{*}\|u-v\| T^{\alpha+\beta-\gamma}}{2 \alpha \Gamma(\alpha-1) \Gamma(\beta)}\left(\frac{1-\gamma}{\beta-\gamma}\right)^{1-\gamma} \int_{0}^{1}(1-\eta)^{\alpha-2} \eta^{\beta-\gamma} d \eta+\frac{2|\lambda| T^{\alpha}}{\Gamma(\alpha+1)}\|u-v\| \\
& \leq\left[\frac{(4 \alpha+\beta-\gamma) \Gamma(\beta-\gamma+1) \mu^{*} T^{\alpha+\beta-\gamma}}{2 \alpha \Gamma(\beta) \Gamma(\alpha+\beta-\gamma+1)}\left(\frac{1-\gamma}{\beta-\gamma}\right)^{1-\gamma}+\frac{2|\lambda| T^{\alpha}}{\Gamma(\alpha+1)}\right]\|u-v\| \\
& =\Lambda\|u-v\| .
\end{aligned}
$$

Since $\Lambda<1$, consequently $F$ is a contraction. As a consequence of Banach fixed point theorem, we deduce that $F$ has a fixed point which is a solution of problem (1.1).

Corollary 3.2. Assume that

$(H 1)^{\prime}$ There exists a constant $L>0$ such that

$$
|f(t, u)-f(t, v)| \leq L|u-v|, \quad \forall t \in[0, T], u, v \in R
$$




$$
\frac{(4 \alpha+\beta) L T^{\alpha+\beta}}{2 \alpha \Gamma(\alpha+\beta+1)}+\frac{2|\lambda| T^{\alpha}}{\Gamma(\alpha+1)}<1
$$

then problem (1.1) has a unique solution.

Theorem 3.3. Suppose that (H1) and the following condition hold:

(H2) There exists a constant $l \in(0,1)$ and a real-valued function $m(t) \in L^{1 / l}\left([0, T], R^{+}\right)$such that

$$
|f(t, u)| \leq m(t), \quad \text { for almost every } t \in[0, T], u \in R
$$

Then the problem (1.1) has at least one solution on $[0, T]$ if

$$
\frac{(2 \alpha+\beta-\gamma) \Gamma(\beta-\gamma+1) \mu^{*} T^{\alpha+\beta-\gamma}}{2 \alpha \Gamma(\beta) \Gamma(\alpha+\beta-\gamma+1)}\left(\frac{1-\gamma}{\beta-\gamma}\right)^{1-\gamma}+\frac{|\lambda| T^{\alpha}}{\Gamma(\alpha+1)}<1
$$

Proof. Let us fix

$$
\frac{(4 \alpha+\beta-l) \Gamma(\beta-l+1) m^{*} T^{\alpha+\beta-l}}{2 \alpha \Gamma(\beta) \Gamma(\alpha+\beta-l+1)\left(1-\left(2|\lambda| T^{\alpha} /(\Gamma(\alpha+1))\right)\right)}\left(\frac{1-l}{\beta-l}\right)^{1-l} \leq r ;
$$

here, $m^{*}=\left(\int_{0}^{T}(m(\tau))^{1 / l} d \tau\right)^{l}$; consider $B_{r}=\{u \in \Omega:\|u\| \leq r\}$, then $B_{r}$ is a closed, bounded, and convex subset of Banach space $\Omega$. We define the operators $S$ and $T$ on $B_{r}$ as

$$
\begin{aligned}
(S u)(t)= & \int_{0}^{t} \frac{(t-s)^{\alpha-1}}{\Gamma(\alpha)}\left(\int_{0}^{s} \frac{(s-\tau)^{\beta-1}}{\Gamma(\beta)} f(\tau, u(\tau)) d \tau-\lambda u(s)\right) d s \\
(T u)(t)= & -\frac{1}{2} \int_{0}^{T} \frac{(T-s)^{\alpha-1}}{\Gamma(\alpha)}\left(\int_{0}^{s} \frac{(s-\tau)^{\beta-1}}{\Gamma(\beta)} f(\tau, u(\tau)) d \tau-\lambda u(s)\right) d s \\
& +\frac{T^{\alpha}-2 t^{\alpha}}{2 \alpha T^{\alpha-1}} \int_{0}^{T} \frac{(T-s)^{\alpha-2}}{\Gamma(\alpha-1)}\left(\int_{0}^{s} \frac{(s-\tau)^{\beta-1}}{\Gamma(\beta)} f(\tau, u(\tau)) d \tau-\lambda u(s)\right) d s .
\end{aligned}
$$

For $u, v \in B_{r}$, based on Hölder inequality, we find that

$$
\begin{aligned}
& |S u+T v| \\
& \leq \int_{0}^{t} \frac{(t-s)^{\alpha-1}}{\Gamma(\alpha)}\left(\int_{0}^{s} \frac{(s-\tau)^{\beta-1}}{\Gamma(\beta)}|f(\tau, u(\tau))| d \tau+|\lambda u(s)|\right) d s \\
& \quad+\frac{1}{2} \int_{0}^{T} \frac{(T-s)^{\alpha-1}}{\Gamma(\alpha)}\left(\int_{0}^{s} \frac{(s-\tau)^{\beta-1}}{\Gamma(\beta)}|f(\tau, v(\tau))| d \tau+|\lambda v(s)|\right) d s
\end{aligned}
$$


Boundary Value Problems

$$
\begin{aligned}
& +\frac{T}{2 \alpha} \int_{0}^{T} \frac{(T-s)^{\alpha-2}}{\Gamma(\alpha-1)}\left(\int_{0}^{s} \frac{(s-\tau)^{\beta-1}}{\Gamma(\beta)}|f(\tau, v(\tau))| d \tau+|\lambda v(s)|\right) d s \\
& \leq \frac{1}{\Gamma(\alpha) \Gamma(\beta)} \int_{0}^{t}(t-s)^{\alpha-1}\left(\int_{0}^{s}(s-\tau)^{\beta-1} m(\tau) d \tau\right) d s+|\lambda|\|u\| \int_{0}^{t} \frac{(t-s)^{\alpha-1}}{\Gamma(\alpha)} d s \\
& +\frac{1}{2 \Gamma(\alpha) \Gamma(\beta)} \int_{0}^{T}(T-s)^{\alpha-1}\left(\int_{0}^{s}(s-\tau)^{\beta-1} m(\tau) d \tau\right) d s+\frac{|\lambda|\|u\|}{2} \int_{0}^{T} \frac{(T-s)^{\alpha-1}}{\Gamma(\alpha)} d s \\
& +\frac{T}{2 \alpha \Gamma(\alpha-1) \Gamma(\beta)} \int_{0}^{T}(T-s)^{\alpha-2}\left(\int_{0}^{s}(s-\tau)^{\beta-1} m(\tau) d \tau\right) d s+\frac{|\lambda| T\|u\|}{2 \alpha} \int_{0}^{T} \frac{(T-s)^{\alpha-2}}{\Gamma(\alpha-1)} d s \\
& \leq \frac{1}{\Gamma(\alpha) \Gamma(\beta)} \int_{0}^{t}(t-s)^{\alpha-1}\left[\left(\int_{0}^{s}\left((s-\tau)^{\beta-1}\right)^{1 /(1-l)} d \tau\right)^{1-l}\left(\int_{0}^{s}(m(\tau))^{1 / l} d \tau\right)^{l}\right] d s \\
& +\frac{1}{2 \Gamma(\alpha) \Gamma(\beta)} \int_{0}^{T}(T-s)^{\alpha-1}\left[\left(\int_{0}^{s}\left((s-\tau)^{\beta-1}\right)^{1 /(1-l)} d \tau\right)^{1-l}\left(\int_{0}^{s}(m(\tau))^{1 / l} d \tau\right)^{l}\right] d s \\
& +\frac{T}{2 \alpha \Gamma(\alpha-1) \Gamma(\beta)} \int_{0}^{T}(T-s)^{\alpha-2}\left[\left(\int_{0}^{s}\left((s-\tau)^{\beta-1}\right)^{1 /(1-l)} d \tau\right)^{1-l}\left(\int_{0}^{s}(m(\tau))^{1 / l} d \tau\right)^{l}\right] d s \\
& +|\lambda|\|u\| \int_{0}^{t} \frac{(t-s)^{\alpha-1}}{\Gamma(\alpha)} d s+\frac{|\lambda|\|u\|}{2} \int_{0}^{T} \frac{(T-s)^{\alpha-1}}{\Gamma(\alpha)} d s+\frac{|\lambda| T\|u\|}{2 \alpha} \int_{0}^{T} \frac{(T-s)^{\alpha-2}}{\Gamma(\alpha-1)} d s \\
& \leq \frac{m^{*}}{\Gamma(\alpha) \Gamma(\beta)}\left(\frac{1-l}{\beta-l}\right)^{1-l} \int_{0}^{t}(t-s)^{\alpha-1} s^{\beta-l} d s \\
& +\frac{m^{*}}{2 \Gamma(\alpha) \Gamma(\beta)}\left(\frac{1-l}{\beta-l}\right)^{1-l} \int_{0}^{T}(T-s)^{\alpha-1} s^{\beta-l} d s \\
& +\frac{m^{*} T}{2 \alpha \Gamma(\alpha-1) \Gamma(\beta)}\left(\frac{1-l}{\beta-l}\right)^{1-l} \int_{0}^{T}(T-s)^{\alpha-2} s^{\beta-l} d s+\frac{2|\lambda| T^{\alpha} r}{\Gamma(\alpha+1)} \\
& \leq \frac{m^{*} T^{\alpha+\beta-l}}{\Gamma(\alpha) \Gamma(\beta)}\left(\frac{1-l}{\beta-l}\right)^{1-l} \int_{0}^{1}(1-\xi)^{\alpha-1} \xi^{\beta-l} d \xi \\
& +\frac{m^{*} T^{\alpha+\beta-l}}{2 \Gamma(\alpha) \Gamma(\beta)}\left(\frac{1-l}{\beta-l}\right)^{1-l} \int_{0}^{1}(1-\eta)^{\alpha-1} \eta^{\beta-l} d \eta \\
& +\frac{m^{*} T^{\alpha+\beta-l}}{2 \alpha \Gamma(\alpha-1) \Gamma(\beta)}\left(\frac{1-l}{\beta-l}\right)^{1-l} \int_{0}^{1}(1-\eta)^{\alpha-2} \eta^{\beta-l} d \eta+\frac{2|\lambda| T^{\alpha} r}{\Gamma(\alpha+1)} \\
& =\frac{(4 \alpha+\beta-l) \Gamma(\beta-l+1) m^{*} T^{\alpha+\beta-l}}{2 \alpha \Gamma(\beta) \Gamma(\alpha+\beta-l+1)}\left(\frac{1-l}{\beta-l}\right)^{1-l}+\frac{2|\lambda| T^{\alpha} r}{\Gamma(\alpha+1)} \\
& \leq r \text {. }
\end{aligned}
$$

Thus, $\|S u+T v\| \leq r$, so $S u+T v \in B_{r}$. 
For $u, v \in \Omega$ and for each $t \in[0, T]$, by the analogous argument to the proof of Theorem 3.1, we obtain

$$
\begin{aligned}
& |(T u)(t)-(T v)(t)| \\
& \leq \frac{1}{2} \int_{0}^{T} \frac{(T-s)^{\alpha-1}}{\Gamma(\alpha)}\left(\int_{0}^{s} \frac{(s-\tau)^{\beta-1}}{\Gamma(\beta)}|f(\tau, u(\tau))-f(\tau, v(\tau))| d \tau\right) d s \\
& +\frac{|\lambda|}{2} \int_{0}^{T} \frac{(T-s)^{\alpha-1}}{\Gamma(\alpha)}|u(s)-v(s)| d s \\
& +\frac{T}{2 \alpha} \int_{0}^{T} \frac{(T-s)^{\alpha-2}}{\Gamma(\alpha-1)}\left(\int_{0}^{s} \frac{(s-\tau)^{\beta-1}}{\Gamma(\beta)}|f(\tau, u(\tau))-f(\tau, v(\tau))| d \tau\right) d s \\
& +\frac{|\lambda| T}{2 \alpha} \int_{0}^{T} \frac{(T-s)^{\alpha-2}}{\Gamma(\alpha-1)}|u(s)-v(s)| d s \\
& \leq \frac{\|u-v\|}{2 \Gamma(\alpha) \Gamma(\beta)} \int_{0}^{T}(T-s)^{\alpha-1}\left(\int_{0}^{s}(s-\tau)^{\beta-1} \mu(\tau) d \tau\right) d s+\frac{|\lambda| T^{\alpha}}{2 \Gamma(\alpha+1)}\|u-v\| \\
& +\frac{T\|u-v\|}{2 \alpha \Gamma(\alpha-1) \Gamma(\beta)} \int_{0}^{T}(T-s)^{\alpha-2}\left(\int_{0}^{s}(s-\tau)^{\beta-1} \mu(\tau) d \tau\right) d s+\frac{|\lambda| T^{\alpha}}{2 \Gamma(\alpha+1)}\|u-v\| \\
& \leq \frac{\|u-v\|}{2 \Gamma(\alpha) \Gamma(\beta)} \int_{0}^{T}(T-s)^{\alpha-1}\left[\left(\int_{0}^{s}\left((s-\tau)^{\beta-1}\right)^{1 /(1-\gamma)} d \tau\right)^{1-\gamma}\left(\int_{0}^{s}(\mu(\tau))^{1 / \gamma} d \tau\right)^{\gamma}\right] d s \\
& +\frac{T\|u-v\|}{2 \alpha \Gamma(\alpha-1) \Gamma(\beta)} \int_{0}^{T}(T-s)^{\alpha-2}\left[\left(\int_{0}^{s}\left((s-\tau)^{\beta-1}\right)^{1 /(1-\gamma)} d \tau\right)^{1-\gamma}\left(\int_{0}^{s}(\mu(\tau))^{1 / \gamma} d \tau\right)^{\gamma}\right] d s \\
& +\frac{|\lambda| T^{\alpha}}{\Gamma(\alpha+1)}\|u-v\| \\
& \leq \frac{\mu^{*}\|u-v\|}{2 \Gamma(\alpha) \Gamma(\beta)}\left(\frac{1-\gamma}{\beta-\gamma}\right)^{1-\gamma} \int_{0}^{T}(T-s)^{\alpha-1} s^{\beta-\gamma} d s \\
& +\frac{\mu^{*} T\|u-v\|}{2 \alpha \Gamma(\alpha-1) \Gamma(\beta)}\left(\frac{1-\gamma}{\beta-\gamma}\right)^{1-\gamma} \int_{0}^{T}(T-s)^{\alpha-2} s^{\beta-\gamma} d s+\frac{|\lambda| T^{\alpha}}{\Gamma(\alpha+1)}\|u-v\| \\
& =\frac{\mu^{*}\|u-v\| T^{\alpha+\beta-\gamma}}{2 \Gamma(\alpha) \Gamma(\beta)}\left(\frac{1-\gamma}{\beta-\gamma}\right)^{1-\gamma} \int_{0}^{1}(1-\eta)^{\alpha-1} \eta^{\beta-\gamma} d \eta \\
& +\frac{\mu^{*}\|u-v\| T^{\alpha+\beta-\gamma}}{2 \alpha \Gamma(\alpha-1) \Gamma(\beta)}\left(\frac{1-\gamma}{\beta-\gamma}\right)^{1-\gamma} \int_{0}^{1}(1-\eta)^{\alpha-2} \eta^{\beta-\gamma} d \eta+\frac{|\lambda| T^{\alpha}}{\Gamma(\alpha+1)}\|u-v\| \\
& \leq\left[\frac{(2 \alpha+\beta-\gamma) \Gamma(\beta-\gamma+1) \mu^{*} T^{\alpha+\beta-\gamma}}{2 \alpha \Gamma(\beta) \Gamma(\alpha+\beta-\gamma+1)}\left(\frac{1-\gamma}{\beta-\gamma}\right)^{1-\gamma}+\frac{|\lambda| T^{\alpha}}{\Gamma(\alpha+1)}\right]\|u-v\| .
\end{aligned}
$$


From the assumption

$$
\frac{(2 \alpha+\beta-\gamma) \Gamma(\beta-\gamma+1) \mu^{*} T^{\alpha+\beta-\gamma}}{2 \alpha \Gamma(\beta) \Gamma(\alpha+\beta-\gamma+1)}\left(\frac{1-\gamma}{\beta-\gamma}\right)^{1-\gamma}+\frac{|\lambda| T^{\alpha}}{\Gamma(\alpha+1)}<1,
$$

it follows that $T$ is a contraction mapping.

The continuity of $f$ implies that the operator $S$ is continuous. Also, $S$ is uniformly bounded on $B_{r}$ as

$$
\|S u\| \leq \frac{\Gamma(\beta-l+1) m^{*} T^{\alpha+\beta-l}}{\Gamma(\beta) \Gamma(\alpha+\beta-l+1)}\left(\frac{1-l}{\beta-l}\right)^{1-l}+\frac{|\lambda| T^{\alpha} r}{\Gamma(\alpha+1)}
$$

On the other hand, let $N=\max _{(t, u) \in[0, T] \times B_{r}}|f(t, u(t))|+1$, for all $\varepsilon>0$, setting

$$
\sigma=\min \left\{\frac{1}{2}\left(\frac{\varepsilon \Gamma(\alpha+\beta)}{2 N}\right)^{1 /(\alpha+\beta)}, \frac{1}{2}\left(\frac{\varepsilon \Gamma(\alpha)}{2|\lambda| r}\right)^{1 / \alpha}\right\}
$$

For each $u \in B_{r}$, we will prove that if $t_{1}, t_{2} \in[0, T]$ and $0<t_{2}-t_{1}<\sigma$, then

$$
\left|(S u)\left(t_{2}\right)-(S u)\left(t_{1}\right)\right|<\varepsilon .
$$

In fact, we have

$$
\begin{aligned}
\mid & (S u)\left(t_{2}\right)-(S u)\left(t_{1}\right) \mid \\
= & \mid \int_{0}^{t_{2}} \frac{\left(t_{2}-s\right)^{\alpha-1}}{\Gamma(\alpha)}\left(\int_{0}^{s} \frac{(s-\tau)^{\beta-1}}{\Gamma(\beta)} f(\tau, u(\tau)) d \tau-\lambda u(s)\right) d s \\
& -\int_{0}^{t_{1}} \frac{\left(t_{1}-s\right)^{\alpha-1}}{\Gamma(\alpha)}\left(\int_{0}^{s} \frac{(s-\tau)^{\beta-1}}{\Gamma(\beta)} f(\tau, u(\tau)) d \tau-\lambda u(s)\right) d s \mid \\
= & \mid \int_{0}^{t_{1}} \frac{\left(t_{2}-s\right)^{\alpha-1}}{\Gamma(\alpha)}\left(\int_{0}^{s} \frac{(s-\tau)^{\beta-1}}{\Gamma(\beta)} f(\tau, u(\tau)) d \tau-\lambda u(s)\right) d s \\
& +\int_{t_{1}}^{t_{2}} \frac{\left(t_{2}-s\right)^{\alpha-1}}{\Gamma(\alpha)}\left(\int_{0}^{s} \frac{(s-\tau)^{\beta-1}}{\Gamma(\beta)} f(\tau, u(\tau)) d \tau-\lambda u(s)\right) d s \\
& -\int_{0}^{t_{1}} \frac{\left(t_{1}-s\right)^{\alpha-1}}{\Gamma(\alpha)}\left(\int_{0}^{s} \frac{(s-\tau)^{\beta-1}}{\Gamma(\beta)} f(\tau, u(\tau)) d \tau-\lambda u(s)\right) d s \mid \\
= & \mid \int_{0}^{t_{1}} \frac{\left(t_{2}-s\right)^{\alpha-1}-\left(t_{1}-s\right)^{\alpha-1}}{\Gamma(\alpha)}\left(\int_{0}^{s} \frac{(s-\tau)^{\beta-1}}{\Gamma(\beta)} f(\tau, u(\tau)) d \tau-\lambda u(s)\right) d s \\
& +\int_{t_{1}}^{t_{2}} \frac{\left(t_{2}-s\right)^{\alpha-1}}{\Gamma(\alpha)}\left(\int_{0}^{s} \frac{(s-\tau)^{\beta-1}}{\Gamma(\beta)} f(\tau, u(\tau)) d \tau-\lambda u(s)\right) d s \mid
\end{aligned}
$$




$$
\begin{aligned}
\leq & \int_{0}^{t_{1}} \frac{\left(t_{2}-s\right)^{\alpha-1}-\left(t_{1}-s\right)^{\alpha-1}}{\Gamma(\alpha)}\left(\int_{0}^{s} \frac{(s-\tau)^{\beta-1}}{\Gamma(\beta)}|f(\tau, u(\tau))| d \tau\right) d s \\
& +|\lambda|\|u\| \int_{0}^{t_{1}} \frac{\left(t_{2}-s\right)^{\alpha-1}-\left(t_{1}-s\right)^{\alpha-1}}{\Gamma(\alpha)} d s \\
& +\int_{t_{1}}^{t_{2}} \frac{\left(t_{2}-s\right)^{\alpha-1}}{\Gamma(\alpha)}\left(\int_{0}^{s} \frac{(s-\tau)^{\beta-1}}{\Gamma(\beta)}|f(\tau, u(\tau))| d \tau\right) d s+|\lambda||| u \| \int_{t_{1}}^{t_{2}} \frac{\left(t_{2}-s\right)^{\alpha-1}}{\Gamma(\alpha)} d s \\
\leq & \frac{N}{\Gamma(\alpha+\beta+1)}\left(t_{2}^{\alpha+\beta}-t_{1}^{\alpha+\beta}\right)+\frac{|\lambda| r}{\Gamma(\alpha+1)}\left(t_{2}^{\alpha}-t_{1}^{\alpha}\right) .
\end{aligned}
$$

In the following, the proof is divided into two cases.

Case 1. For $\sigma \leq t_{1}<t_{2}<T$, we have

$$
\begin{aligned}
\left|(S u)\left(t_{2}\right)-(S u)\left(t_{1}\right)\right| \leq & \frac{N}{\Gamma(\alpha+\beta+1)}\left(t_{2}^{\alpha+\beta}-t_{1}^{\alpha+\beta}\right)+\frac{|\lambda| r}{\Gamma(\alpha+1)}\left(t_{2}^{\alpha}-t_{1}^{\alpha}\right) \\
& \leq \frac{N}{\Gamma(\alpha+\beta+1)}(\alpha+\beta) \sigma^{\alpha+\beta-1}\left(t_{2}-t_{1}\right)+\frac{|\lambda| r}{\Gamma(\alpha+1)} \alpha \sigma^{\alpha-1}\left(t_{2}-t_{1}\right) \\
& <\frac{N}{\Gamma(\alpha+\beta)} \sigma^{\alpha+\beta}+\frac{|\lambda| r}{\Gamma(\alpha)} \sigma^{\alpha} \\
& <\left(\frac{1}{2}\right)^{\alpha+\beta} \frac{\varepsilon}{2}+\left(\frac{1}{2}\right)^{\alpha} \frac{\varepsilon}{2} \\
& <\varepsilon .
\end{aligned}
$$

Case 2. for $0 \leq t_{1}<\sigma, t_{2}<2 \sigma$, we have.

$$
\begin{aligned}
\left|(S u)\left(t_{2}\right)-(S u)\left(t_{1}\right)\right| & \leq \frac{N}{\Gamma(\alpha+\beta+1)}\left(t_{2}^{\alpha+\beta}-t_{1}^{\alpha+\beta}\right)+\frac{|\lambda| r}{\Gamma(\alpha+1)}\left(t_{2}^{\alpha}-t_{1}^{\alpha}\right) \\
& \leq \frac{N}{\Gamma(\alpha+\beta+1)} t_{2}^{\alpha+\beta}+\frac{|\lambda| r}{\Gamma(\alpha+1)} t_{2}^{\alpha} \\
& <\frac{N}{\Gamma(\alpha+\beta+1)}(2 \sigma)^{\alpha+\beta}+\frac{|\lambda| r}{\Gamma(\alpha+1)}(2 \sigma)^{\alpha} \\
& <\frac{\varepsilon}{2}+\frac{\varepsilon}{2} \\
& =\varepsilon .
\end{aligned}
$$

Therefore, $S$ is equicontinuous and the Arzela-Ascoli theorem implies that $S$ is compact on $B_{r}$, so the operator $S$ is completely continuous. 
Thus, all the assumptions of Lemma 2.7 are satisfied and the conclusion of Lemma 2.7 implies that the boundary value problem (1.1) has at least one solution on $[0, T]$.

Corollary 3.4. Suppose that the condition $(\mathrm{H1})^{\prime}$ hold and, assume that

$$
\frac{(2 \alpha+\beta) L T^{\alpha+\beta}}{2 \alpha \Gamma(\alpha+\beta+1)}+\frac{|\lambda| T^{\alpha}}{\Gamma(\alpha+1)}<1 .
$$

Further assume that

(H2)' there exists a constant $K>0$ such that

$$
|f(t, u)| \leq K, \quad \forall t \in[0, T], u \in R,
$$

then problem (1.1) has at least one solution on $[0, T]$.

\section{Example}

Let $\alpha=2, \beta=1, \lambda=1 / 8, T=\pi / 2$. We consider the following boundary value problem

$$
\begin{gathered}
{ }^{C} D^{1}\left({ }^{C} D^{2}+\frac{1}{8}\right) u(t)=f(t, u(t)), \quad 0 \leq t \leq \frac{\pi}{2}, \\
u(0)+u\left(\frac{\pi}{2}\right)=0, \quad u^{\prime}(0)=u^{\prime}\left(\frac{\pi}{2}\right)=0
\end{gathered}
$$

where

$$
f(t, u)=\frac{1}{(t+2)^{2}} \frac{u}{1+u}, \quad(t, u) \in[0, T] \times[0, \infty)
$$

Because of $|f(t, u)-f(t, v)| \leq(1 / 4)|u-v|$, let $\mu(t) \equiv 1 / 4$, then $\mu(t) \in L^{2}([0, \pi / 2])$, we have $\gamma=1 / 2$ and $\mu^{*}=\left(\int_{0}^{T}(\mu(\tau))^{1 / \gamma} d \tau\right)^{\gamma}=\left(\int_{0}^{\pi / 2}(1 / 4)^{2} d \tau\right)^{1 / 2}=\sqrt{\pi} / 4 \sqrt{2}$. Further,

$$
\begin{aligned}
& \frac{(4 \alpha+\beta-\gamma) \Gamma(\beta-\gamma+1) \mu^{*} T^{\alpha+\beta-\gamma}}{2 \alpha \Gamma(\beta) \Gamma(\alpha+\beta-\gamma+1)}\left(\frac{1-\gamma}{\beta-\gamma}\right)^{1-\gamma}+\frac{2|\lambda| T^{\alpha}}{\Gamma(\alpha+1)} \\
& =\frac{(17 / 2) \Gamma(3 / 2) \mu^{*} T^{5 / 2}}{4 \Gamma(1) \Gamma(7 / 2)}+\frac{2|\lambda| T^{2}}{\Gamma(3)} \\
& =\frac{17 \pi^{3}}{15 \times 64}+\frac{\pi^{2}}{32} \\
& \approx 0.86<1 .
\end{aligned}
$$

Then BVP (4.1) has a unique solution on $[0, \pi / 2]$ according to Theorem 3.1. 
On the other hand, we find that

$$
\begin{aligned}
& \frac{(2 \alpha+\beta-\gamma) \Gamma(\beta-\gamma+1) \mu^{*} T^{\alpha+\beta-\gamma}}{2 \alpha \Gamma(\beta) \Gamma(\alpha+\beta-\gamma+1)}\left(\frac{1-\gamma}{\beta-\gamma}\right)^{1-\gamma}+\frac{|\lambda| T^{\alpha}}{\Gamma(\alpha+1)} \\
& =\frac{(9 / 2) \Gamma(3 / 2) \mu^{*} T^{5 / 2}}{4 \Gamma(7 / 2)}+\frac{|\lambda| T^{2}}{\Gamma(3)} \\
& =\frac{9 \pi^{3}}{64 \times 15}+\frac{\pi^{2}}{64} \\
& \approx 0.44<1 .
\end{aligned}
$$

Then BVP (4.1) has at least one solution on $[0, \pi / 2]$ according to Theorem 3.3.

\section{Acknowledgments}

This work was supported by the Natural Science Foundation of China (10971173), the Natural Science Foundation of Hunan Province (10JJ3096), the Aid Program for Science and Technology Innovative Research Team in Higher Educational Institutions of Hunan Province, and the Construct Program of the Key Discipline in Hunan Province.

\section{References}

[1] A. Alsaedi, "Existence of solutions for integrodifferential equations of fractional order with antiperiodic boundary conditions," International Journal of Differential Equations, vol. 2009, Article ID 417606, 9 pages, 2009.

[2] B. Ahmad, "Existence of solutions for fractional differential equations of order $q \in[2,3)$ with antiperiodic boundary conditions," Journal of Applied Mathematics and Computing, vol. 34, no. 1-2, pp. 385-391, 2010.

[3] B. Ahmad and V. Otero-Espinar, "Existence of solutions for fractional differential inclusions with antiperiodic boundary conditions," Boundary Value Problems, vol. 2009, Article ID 625347, 11 pages, 2009.

[4] Z. Bai and H. Lü, "Positive solutions for boundary value problem of nonlinear fractional differential equation," Journal of Mathematical Analysis and Applications, vol. 311, no. 2, pp. 495-505, 2005.

[5] C. F. Li, X. N. Luo, and Y. Zhou, "Existence of positive solutions of the boundary value problem for nonlinear fractional differential equations," Computers $\mathcal{E}$ Mathematics with Applications, vol. 59, no. 3, pp. 1363-1375, 2010.

[6] V. Lakshmikantham, S. Leela, and J. V. Devi, Theory of Fractional Dynamic Systems, Cambridge Academic, Cambridge, UK, 2009.

[7] Y. Tian and A. Chen, "The existence of positive solution to three-point singular boundary value problem of fractional differential equation," Abstract and Applied Analysis, vol. 2009, Article ID 314656, 18 pages, 2009.

[8] S. Zhang, "Positive solutions for boundary-value problems of nonlinear fractional differential equations," Electronic Journal of Differential Equations, vol. 2006, pp. 1-12, 2006.

[9] B. Ahmad and J. J. Nieto, "Solvability of nonlinear Langevin equation involving two fractional orders with Dirichlet boundary conditions," International Journal of Differential Equations, vol. 2010, Article ID 649486, 10 pages, 2010.

[10] B. Ahmad and P. Eloe, "A nonlocal boundary value problem for a nonlinear fractional differential equation with two indices," Communications on Applied Nonlinear Analysis, vol. 17, no. 3, pp. 69-80, 2010. 
[11] S. C. Lim, M. Li, and L. P. Teo, "Langevin equation with two fractional orders," Physics Letters A, vol. 372, no. 42, pp. 6309-6320, 2008.

[12] S. C. Lim and L. P. Teo, "The fractional oscillator process with two indices," Journal of Physics A, vol. 42, Article ID 065208, 34 pages, 2009. 